\title{
Overexpression of the growth arrest-specific homeobox gene Gax inhibits proliferation, migration, cell cycle progression, and apoptosis in serum-induced vascular smooth muscle cells
}

\author{
H. Zheng, S. Xue, Z.L. Hu, J.G. Shan and W.G. Yang \\ Department of Cardiovascular Surgery, Renji Hospital, Shanghai Jiao Tong \\ University School of Medicine, Shanghai, China \\ Corresponding author: S. Xue \\ E-mail: xuesong64@163.com
}

Genet. Mol. Res. 13 (1): 1993-2008 (2014)

Received December 10, 2012

Accepted May 15, 2013

Published March 24, 2014

DOI http://dx.doi.org/10.4238/2014.March.24.4

\begin{abstract}
The Gax gene has been implicated in a variety of celldevelopmental and biological processes, and aberrant Gax expression is linked to many diseases. In this study, to provide important insights for Gax-based gene therapy in vein graft restenosis and its anti-restenotic mechanism, we used rabbit vascular smooth muscle cells (VSMCs) to investigate the effects of Gax overexpression on proliferation, migration, cell cycle, and apoptosis in a serum-stimulated culture. Rabbit VSMC lines that stably overexpressed Gax were established by transfection with recombinant adenoviral vector Ad5-Gax. The effect of Gax overexpression on in vitro serum-induced VSMCs proliferation, migration, cell cycle, and apoptosis was assessed by MTT, wound healing, and flow cytometry assays, respectively. To investigate the effect of Gax overexpression on PCNA and MMP-2 in serum-induced VSMCs, immunocytochemistry, RT-PCR, and gelatin zymography
\end{abstract}


were performed. The results clearly showed that Gax overexpression decreases PCNA expression in serum-induced VSMCs. Gax overexpression also significantly inhibited cell proliferation by blocking entry into the S-phase of the cell cycle, promoted cell apoptosis, and reduced cell migration activity by downregulating MMP-2 release and activity. These findings indicate that Gax would be an optimal target gene for gene therapy to treat vein graft restenosis.

Key words: Apoptosis; Cell cycle; Gax; Migration; VSMCs; Proliferation

\section{INTRODUCTION}

Altered Gax gene expression is associated with the development of many diseases, including vascular restenosis, Alzheimer disease, Hutchinson-Gilford progeria syndrome (Csoka et al., 2004), cancer, and cleft palate (Li and Ding, 2007). The Gax gene, also called MEOX2, is located in a gene cluster on human chromosome 7P21, and codes for a transcription factor, which is predominantly expressed in mesoderm and muscle precursors in the embryo and in the cardiovascular system of adults (Candia and Wright, 1996; Skopicki et al., 1997). Gax has been implicated in cellular differentiation and the control of body plan formation by regulating the expression of a number of genes whose products function as central regulators. Overexpression of Gax with adenoviral vectors in vitro can inhibit TGF- $\beta 1$-induced adventitial fibroblast proliferation, migration, and adherence; Gax overexpression also decreases the number of S phase cells and increases the number of G0-G1 phase cells and promotes cell apoptosis (Liu et al., 2008). Gax is expressed in quiescent vascular endothelial cells and strongly inhibits endothelial cells activation during stimulation with growth factors or tube formation by activating $\mathrm{p} 21^{\mathrm{WAF} / \mathrm{CIP} 1}$ expression and reducing anti-apoptotic, NF-kB-dependent gene expression, suggesting that Gax is a negative regulator of angiogenesis (Gorski and Leal, 2003; Patel et al., 2005; Chen et al., 2007). In addition, Gax has been shown to be induced at the onset of cellular senescence in keratinocytes (Irelan et al., 2009). In human lung adenocarcinoma A549 cells, Gax overexpression enhanced cell apoptosis and inhibited cell proliferation (Xia et al., 2007). These data led to the suggestion that Gax functions as a negative regulator of cellular processes. However, the role of Gax in serum-induced VSMCs is not entirely clear.

Coronary artery disease, a common post-birth heart disease, affects millions of individuals worldwide, and coronary artery bypass grafting (CABG) is a highly effective procedure for relieving the signs and symptoms of patients with coronary artery disease. Autologous saphenous vein grafts are the most frequently used conduits in CABG surgery. Vein graft restenosis results in a 20 to $50 \%$ failure rate of CABG within 10 years (Veith et al., 1986; Fitzgibbon et al., 1996) seriously affecting the long-term benefits of CABG. Neointimal hyperplasia is a key pathological process resulting in the occlusion of the grafted veins and is characterized by abnormal proliferation of VSMCs, increased migration from the media toward the intima, and reduced apoptosis (Mitra et al., 2006). However, no effective interventions for vein graft failure are available to date. With the development of molecular biology-based techniques, gene therapy now offers a potential therapeutic alternative. In this approach, efficient expression of the therapeutic transgene is required. Many studies have reported that Gax expression 
in response to mitogens is downregulated in VSMCs. However, relatively little is known about the effects of increased expression of Gax on the biological behaviors in VSMCs. In this study, we constructed a recombinant adenoviral vector containing $G a x$, which was transfected into VSMCs for Gax overexpression and investigated in vitro effects of Gax overexpression on biological behaviors of serum-induced VSMCs. In particular, we were interested in the potential use of Gax as a candidate gene for gene therapy to treat vein graft restenosis.

\section{MATERIAL AND METHODS}

\section{Cell culture}

Experiments were conducted in full compliance with the Animal Ethical Committee of the Medical School, Shanghai Jiaotong University. Primary cultures of rabbit vascular smooth muscle cells (VSMCs) were prepared from thoracic aortas of adult male New Zealand White rabbits and cultured in Dulbecco's modified Eagle's medium (DMEM) (Gibco, USA), supplemented with 10\% fetal bovine serum (FBS) (Gibco) and penicillin $(100 \mathrm{U} / \mathrm{mL}) /$ streptomycin $(100 \mathrm{U} / \mathrm{mL})$. The cells were cultured in an incubator at $37^{\circ} \mathrm{C}$ under $5 \% \mathrm{CO}_{2}$. VSMCs from the 3 rd to 5 th generation were used for follow-up studies.

\section{Construction of recombinant Gax adenoviral vector}

The pDC318-mCMV shuttle plasmid, pPE3 skeleton plasmid, and Ad5-EGFP were a generous gift from Dr. Qian Qi-jun. pEGFP-N1-Gax vector was digested with restriction endonuclease NheI and HindIII. The digestion product was electrophoresed on $1.5 \%$ agarose gel at $10 \mathrm{~V} / \mathrm{cm}$, and examined by using a UV-transmission reflection-type analyzer. The Gax gene (DNA fragment size, $927 \mathrm{bp}$ ) was extracted and purified. The purified Gax gene was ligated with T4 DNA ligase into the digested pDC318-mCMV vector to construct the vector pDC318mCMV-Gax. The ligation mixture was transformed into DH5 $\alpha$ competent cells. Ampicillin-resistant transformants were selected by plating the transformation mixture on LB agar plates with $50 \mu \mathrm{g} / \mathrm{ml}$ ampicillin. After incubation overnight, the recombinant plasmid DNA was isolated by the alkaline lysis method. Double digestion and DNA sequencing were used to verify that the desired construct was obtained, which was named pDC318-mCMV-Gax. The pDC318-mCMVGax vector was then cotransfected with pPE3 vector into 293 cells by using Lipofectamine 2000 to obtain the replication-defective recombinant adenoviral vector containing the Gax gene, confirmed by PCR. The resulting recombinant adenoviral vector was named Ad5-Gax. After amplification and purification, the Ad5-Gax vector was used to transfect VSMCs.

\section{Gene transfection}

VSMCs were seeded into 6-well plates in DMEM containing 10\% FBS and grown to $70-80 \%$ confluence at $37^{\circ} \mathrm{C}$ overnight. The medium was transferred to DMEM without FBS. Ad5-Gax or Ad5-EGFP was added to the medium at a multiplicity of infection (MOI) of 30. After incubation for 4 hours, the virus was removed by washing 3 times with PBS, and returned to DEME without FBS for incubation at an additional $12 \mathrm{~h}$. The cells were then stimulated by the addition of $10 \%$ FBS. 


\section{RT-PCR analysis}

TRIzol reagent (Invitrogen) was used to extract total cellular RNA at days 1, 3, 5, and 7 after gene transfection. Single-stranded cDNA was synthesized using a reverse-transcription system kit (Invitrogen). The reaction included $4 \mu \mathrm{L} \mathrm{MgCl}_{2}, 4 \mu \mathrm{L}$ buffer, $1 \mu \mathrm{L}$ oligo-dT, $2 \mu \mathrm{L}$ dNTP, $2 \mu \mathrm{g}$ RNA, $0.5 \mu \mathrm{L}$ RNase, $0.5 \mu \mathrm{L}$ reverse transcriptase, and $\mathrm{ddH}_{2} \mathrm{O}$ added to the sample to a final volume of $20 \mu \mathrm{L}$. The reverse-transcription reaction was performed at $30^{\circ} \mathrm{C}$ for 10 $\min , 42^{\circ} \mathrm{C}$ for $60 \mathrm{~min}, 99^{\circ} \mathrm{C}$ for $5 \mathrm{~min}$, and $5^{\circ} \mathrm{C}$ for $5 \mathrm{~min}$. The amplification for Gax/MMP2 was performed under the following conditions: a $20-\mu \mathrm{L}$ reaction mixture contained $2 \mu \mathrm{L}$ PCR buffer, $1.2 \mu \mathrm{L} \mathrm{MgCl}_{2}, 0.5 \mu \mathrm{L}$ dNTP, $1 \mu \mathrm{L}$ cDNA, $0.25 \mu \mathrm{L} 5 \mathrm{U} / \mu \mathrm{L}$ Taq polymerase, 0.3 $\mu \mathrm{L}$ of each primer (Gax sense: 5'-ACCACCATCACCACCATCATC-3' and Gax anti-sense: 5'-TGGAAGAGTTGGAGCACAGG-3'; MMP-2 sense: 5'-ACAGGATCATCGGCTACACG-

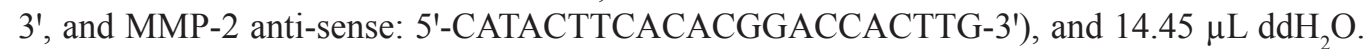
The cycling conditions were the following: 1 cycle of $95^{\circ} \mathrm{C}$ for $3 \mathrm{~min}$, followed by 35 cycles of $95^{\circ} \mathrm{C}$ for $45 \mathrm{~s}, 55^{\circ} \mathrm{C}$ for $1 \mathrm{~min}$, and $72^{\circ} \mathrm{C}$ for $2 \mathrm{~min}$; a final hold was at $72^{\circ} \mathrm{C}$ for $10 \mathrm{~min}$. The gene for glyceraldehyde-3-phosphate dehydrogenase $(G A P D H)$ was used as an internal standard. PCR products were electrophoresed on $1.5 \%$ agarose gelatin.

\section{Immunofluorescence}

Rabbit VSMCs were seeded into the 6-well plates in growth media. At days 1, 3, 5, and 7 after viral infection, the cells were fixed for 20 min with $4 \%$ neutral buffered formalin and permeabilized with $0.1 \%$ Triton X-100 for $10 \mathrm{~min}$. The VSMCs were blocked for $30 \mathrm{~min}$ in $10 \%$ goat serum before addition of the primary antibody. A 1:400 dilution of anti-Gax antibody was incubated overnight at $4^{\circ} \mathrm{C}$. After multiple washes with PBS, the cells were incubated with the FITC-conjugated goat anti-mouse secondary antibody (diluted 1:1000) (Molecular Probes) for 1 h. VSMCs were rinsed with PBS three times and visualized by fluorescent microscopy.

\section{Cell proliferation assay}

VSMCs were cultured in 96-well plates at $8 \times 10^{3}$ cells/well; $20 \mu \mathrm{L}$ of a $5 \mathrm{mg} / \mathrm{mL}$ MTT solution (Sigma) was added to the cell cultures at various time points $(24,48,72$, and $96 \mathrm{~h}$ after gene transfection) using 6 wells for each group per time point. The plates were incubated at $37^{\circ} \mathrm{C}$ in air with $5 \% \mathrm{CO}_{2}$ for $4 \mathrm{~h}$. The reaction was terminated by adding $150 \mu \mathrm{L}$ DMSO per well and the VSMCs were lysed for 10 min. Optical density was determined at $570 \mathrm{~nm}$ wavelength in an enzyme-linked immunosorbent assay reader (Thermo, Varioskan 2.2, USA). The inhibition rate was determined as (1 - average OD of experimental well/average OD of blank control well) x $100 \%$.

\section{Cell migration assay}

VSMCs were seeded to $90 \%$ confluence on 6-well plates. A 1-mL sterile pipet tip was used to scratch a central linear track (or "wound") into the well, and floating cells were removed by changing the culture media. Phase micrographs of the track were taken at $0,24,48$, and $72 \mathrm{~h}$ after gene transfection. The track width was measured at all time points. The cell migration activity was 
counted as the mean wound width between edges of 3 points in 10 randomly selected fields per well. The rate of cell migration was calculated as (mean original scratch width - mean scratch width at a time point)/ mean original scratch width $\mathrm{x} 100 \%$. Each experiment was performed in triplicate.

\section{Cell cycle analysis}

Rabbit VSMCs were treated with trypsin to yield single-cell suspensions and fixed in cold $70 \%$ ethanol at $4{ }^{\circ} \mathrm{C}$ for $30 \mathrm{~min}$. After washing with PBS, the cells were incubated in a solution containing $50 \mathrm{ng} / \mathrm{mL}$ propidium iodide, $0.2 \mathrm{mg} / \mathrm{mL}$ RNase, and $0.1 \%$ Triton X-100 at room temperature for $30 \mathrm{~min}$ in the dark. The cells were then detected by flow cytometry using an EPICS ALTRA FACScan (Beckman Coulter, USA). The data were analyzed with the CXP software.

\section{Apoptosis assay}

To detect apoptosis after gene transfection, the Annexin V-FITC Apoptosis Detection Kit (Beyotime, Nanjing, China) was used. Briefly, rabbit VSMCs were trypsinized and washed once with PBS. Then the cells were stained with Annexin V/FITC for $30 \mathrm{~min}$ at $4^{\circ} \mathrm{C}$ and propidium iodide for $15 \mathrm{~min}$ in the dark according to manufacturer protocol. The rate of cell apoptosis was determined by flow cytometry.

\section{DAPI staining}

A DAPI staining kit (Beyotime, Nanjing, China) was used to confirm morphological changes in the nuclei. VSMCs were seeded in 6-well plates, infected as described above, fixed in $4 \%$ neutral buffered formalin, washed twice with PBS, and stained with DAPI-staining solution for $3 \mathrm{~min}$ at room temperature in the dark. After 2 washes, the VSMCs were evaluated and images were captured using an Olympus IX70-SIF2 fluorescence microscope with a UV filter. For each group, 15 shots were randomly selected to determine the percentage of apoptotic cells in the total number of cells. Apoptotic cells were defined by deep-staining nuclear condensation of nuclear chromatin and fragmentation of the nuclear membrane.

\section{Immunocytochemistry}

The expression of PCNA was detected by immunoperoxidase staining. VSMCs were cultured in 6-well plates and infected as described above. The cells were fixed, washed twice with PBS, and permeabilized with $0.1 \%$ Triton X-100 for 10 min. A 1:500 dilution of antibody against PCNA was incubated overnight at $4^{\circ} \mathrm{C}$. After 3 washes, the cells were incubated with HRP-labeled secondary antibody (1:1000) for $1 \mathrm{~h}$. VSMCs were then examined under the microscope for color development after reaction with $\mathrm{DAB}$ reagent. The nucleus were stained by hematoxylin. The cells were visualized and photographed under an inverted microscope, and 15 shots were randomly selected to determine the ratio of PCNA-expressing VSMCs.

\section{Gelatin zymography}

MMP-2 activity in the DMEM medium was determined by gelatin zymography. Equal 
volumes of the aforementioned medium conditioned from serum-induced VSMCs transfected with Ad5-Gax, Ad5-EGFP, or nontransfected (in PBS) were loaded onto SDS-PAGE gels containing $1 \mathrm{mg} / \mathrm{mL}$ gelatin (Sigma). After the electrophoresis, in order to renature the proteins, the gels were washed twice in $2.5 \%$ Triton X-100 for 15 minutes at room temperature to remove SDS. Next, the gels were incubated in a substrate buffer $(50 \mathrm{mM}$ Tris- $\mathrm{HCl}$ and $5 \mathrm{mM}$ $\mathrm{CaCl}_{2}, \mathrm{pH} 7.5$ ) overnight at $37^{\circ} \mathrm{C}$. Then, the gels were stained with $0.1 \%$ Coomassie brilliant blue R-250 in 20\% ethanol and 10\% acetic acid, followed by destaining in $20 \%$ ethanol/10\% acetic acid/water until clear protein bands appeared. Clear bands against the background indicated the presence of gelatinolytic activity. The gelatinolytic bands were densitometrically scanned with the gel-imaging system Tanon-2500.

\section{Statistical analysis}

Data are reported as means \pm standard deviation (SD). One-way ANOVA was used for determining statistical significance of variation among different groups with the SPSS 11.0 software. A P $<0.05$ was considered statistically significant.

\section{RESULTS}

\section{Construction and transfection with the recombinant adenoviral vector Ad5-Gax}

To detect the effect of Gax on serum-induced VSMCs in vitro, we constructed the Gax-expression vector Ad5-Gax designed to increase the level of Gax protein in the cells. Ad5-Gax-transfected cells are referred to as VSMC/Gax, and negative-control cells transfected with Ad5-EGFP as VSMC/EGFP; blank control cells were mock transfected in PBS and are referred to as VSMC/PBS. Gax mRNA and protein levels in VSMCs were confirmed by RT-PCR with Gax-specific primers and by immunofluorescence with antiGax antibody, respectively. A conspicuous 174-bp band was observed following 1.5\% gel electrophoresis of PCR products amplified from cellular RNA extracted at days 1, 3, 5, and 7 from VSMC/Gax cell cultures (Figure 1), whereas this band was not detected in VSMC/ EGFP or VSMC/PBS cultures. Moreover, Gax protein expression was also detected on the same days in VSMC/Gax (Figure 2). These results suggested that the recombinant adenovirus vector Ad5-Gax had been successfully constructed and that Gax was stably overexpressed in the VSMC/Gax.

\section{Overexpression of Gax inhibits cell proliferation of serum-induced VSMCs}

To examine the role of Gax in VSMCs postserum stimulation, we first determined the viability of serum-induced VSMCs after Ad5-Gax transfection by using the MTT assay (Figure 3). The individual OD values at 48, 72, and $96 \mathrm{~h}$ showed that the proliferative activity of VSMC/Gax was significantly lower than that of the control cells (Figure 4A; P<0.05). As shown in Figure 4B, VSMC/EGFP had lower mean inhibition rates than VSMCs/Gax and the growth-inhibiting effect of Gax on serum-induced VSMCs increased with extended culture time, which was consistent with the elevated levels of Gax in VSMC/Gax. These data indicated that there was a suppressive effect of Gax on serum-induced VSMCs viability. 


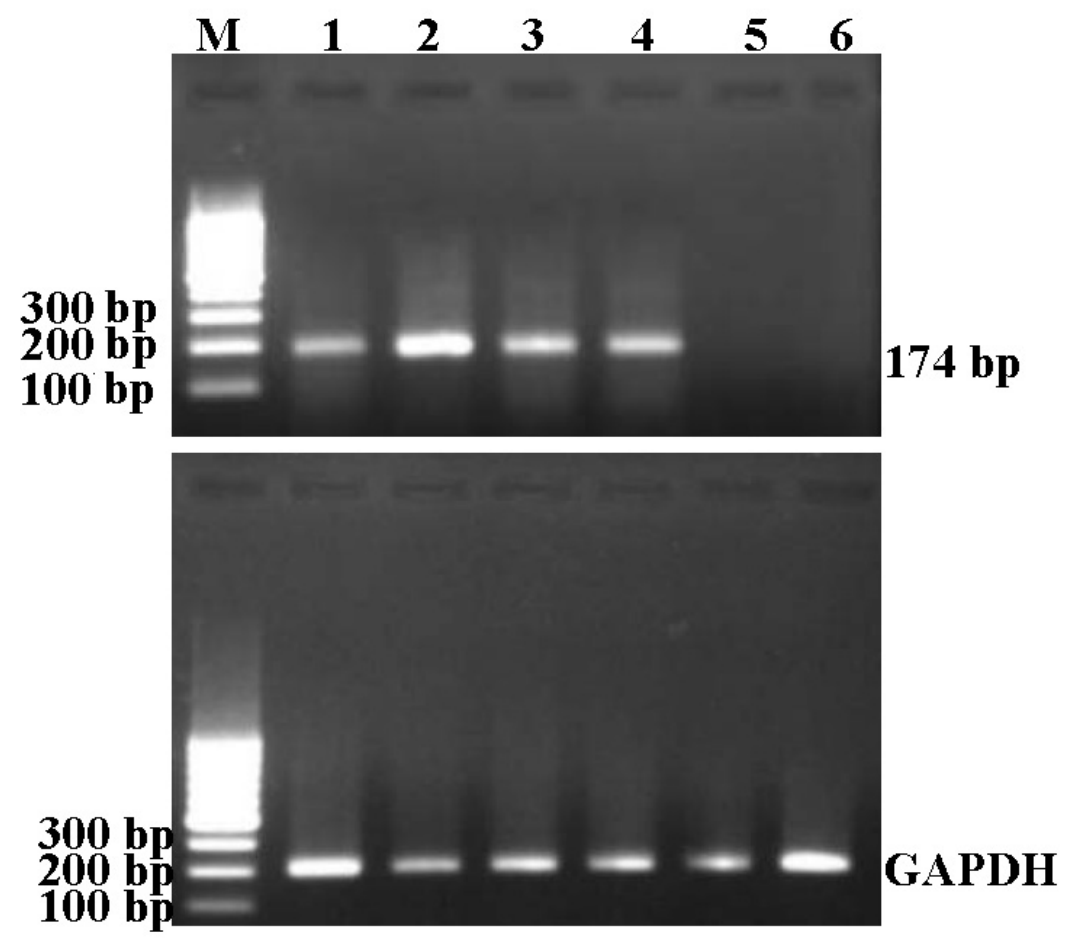

Figure 1. RT-PCR of Gax in Rabbit VSMCs. VSMCs were cultured as described in Section 2. Total cellular RNA was isolated and RT-PCR performed with specific primers for Gax. GAPDH was employed as a internal control. PCR Products in VSMCs/Gax,VSMCs/EGFP and VSMCs/PBS were separated on 1.5\% agarose Gels and stained with ethidium bromide. An obvious Gax band of $174 \mathrm{bp}$ was amplified in VSMCs/Gax and there was no specific band in VSMCs/EGFP and VSMCs/ PBS. Lane $M=\mathrm{DNA}$ marker; lane $1=\mathrm{VSMCs} / \mathrm{Gax}$ at 1 st day after gene transfection; lane $2=\mathrm{VSMCs} / \mathrm{Gax}$ at $3 \mathrm{rd}$ day after gene transfection; lane $3=\mathrm{VSMCs} / \mathrm{Gax}$ at 5 th day after gene transfection; lane $4=\mathrm{VSMCs} / \mathrm{Gax}$ at 7 th day after gene transfection; lane $5=\mathrm{VSMCs} / \mathrm{EGFP}$; lane 6 $=\mathrm{VSMCs} / \mathrm{PBS}$.

A $\mathrm{B}$ $\mathrm{C}$ $\mathrm{D}$

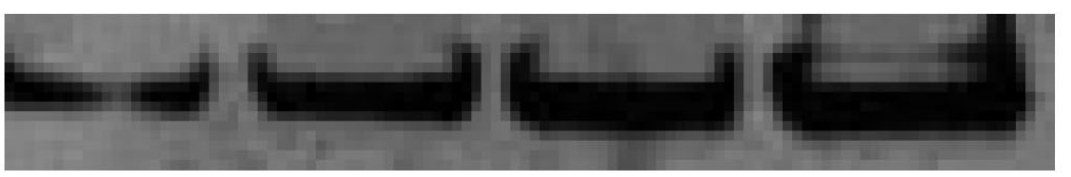

Gax $37 \mathrm{kDa}$

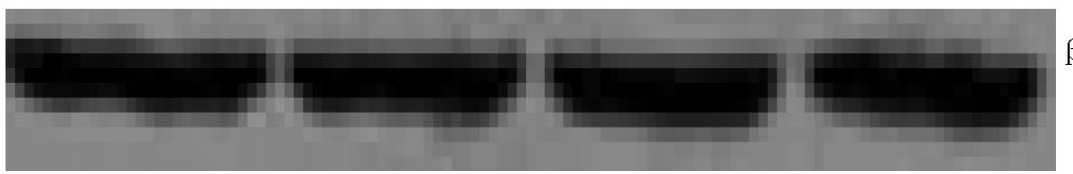

$\beta-$ Actin

Figure 2. Western blot analysis of Gax protein levels in Rabbit VSMCs. Total cellular proteins were extracted and the expression of Gax protein was analyzed using Western blot. $\beta$-Actin was used as a control. (A) VSMCs/Gax at 1st day after gene transfection; (B) VSMCs/Gax at 3rd day after gene transfection; (C) VSMCs/Gax at 5th day after gene transfection; (D) VSMCs/Gax at 7th day after gene transfection. 

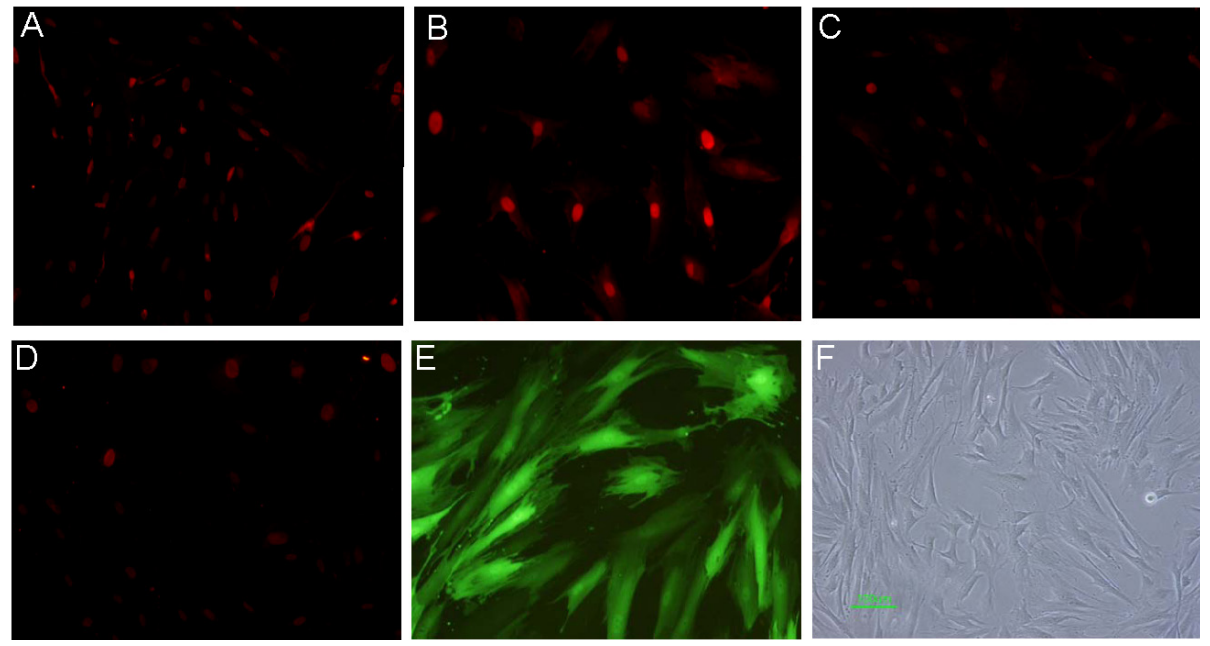

Figure 3. Immunofluorescence staining results of Gax protein in Rabbit VSMC. The cells were grown and transfected with Ad5-Gax, Ad5-EGFP, or PBS. After that, the cells were stained and reviewed under a fluorescent microscopy. A. Ad5-Gax-transfected VSMCs at 1st day (100X); B. Ad5-Gax-transfected VSMCs at 3rd day (100X); C. Ad5-Gax-transfected VSMCs at 5th day (100X); D. Ad5-Gax-transfected VSMCs at 7th day (100X); E. Ad5-EGFP-transfected VSMCs (100X); F. PBS-transfected VSMCs under the inverted microscopy (100X).

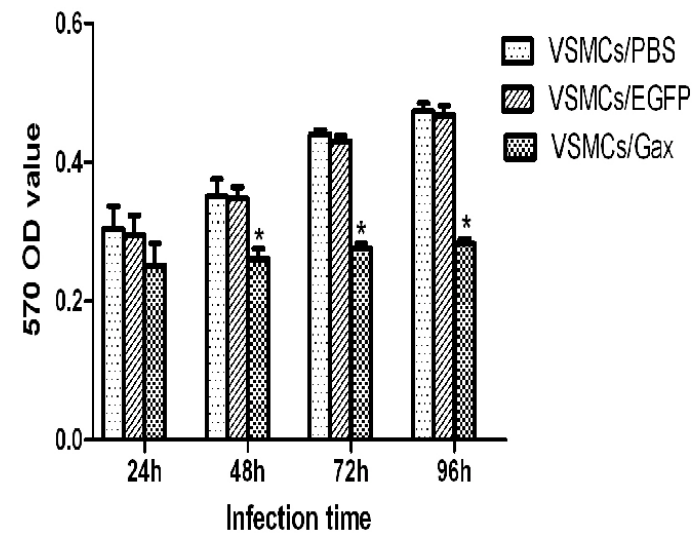

A

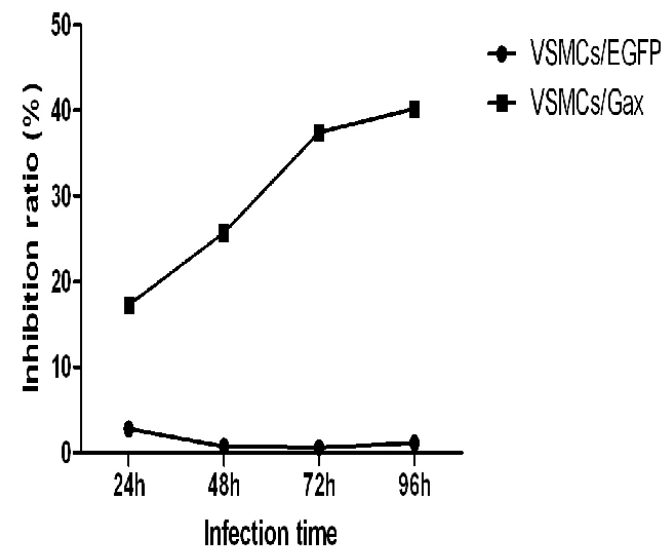

B

Figure 4. VSMCs viability was assessed by MTT. The results are reported as means \pm SD from 5 independent experiments. A. Cells viability under serum stimulation was observed in VSMCs/ Gax, VSMCs/EGFP, VSMCs/ PBS at 24, 48, 72, $96 \mathrm{~h}$, based on the results of MTT assays, ${ }^{*} \mathrm{P}<0.05 v s$ VSMCs/PBS or VSMCs/EGFP; B. The mean cell inhibition rate was calculated at $24,48,72,96 \mathrm{~h}$ in VSMCs/Gax and VSMCs/EGFP.

\section{Effect of Gax overexpression on serum-induced VSMCs migration}

We measured the migration ability of VSMC/Gax using the wound-healing assay. A 
central linear wound in the monolayer VSMCs was made. The width of the wound, measured as the distance between its edges, was examined at $0 \mathrm{~h}, 24 \mathrm{~h}, 48 \mathrm{~h}$, and $72 \mathrm{~h}$ of incubation. VSMC/ Gax exhibited decreased migration activity at all time points compared with VSMC/EGFP and VSMC/PBS $(\mathrm{P}<0.05)$, and there was no statistically significant difference between VSMC/ EGFP and VSMC/PBS. The healing rate in VSMC/Gax at 24, 48, and $72 \mathrm{~h}$ of incubation was $16.23 \pm 4.01,20.12 \pm 3.61$, and $24.87 \pm 3.04 \%$, respectively. In contrast, the corresponding healing rates were $41.86 \pm 1.43,49.47 \pm 4.50$, and $63.58 \pm 1.82 \%$ in VSMCs/PBS and $41.01 \pm$ $2.56,48.64 \pm 2.64$, and $62.41 \pm 2.08 \%$ in VSMCs/EGFP (Figure 5). Therefore, it is suggested that Gax expression reduces the migration of serum-induced VSMCs.
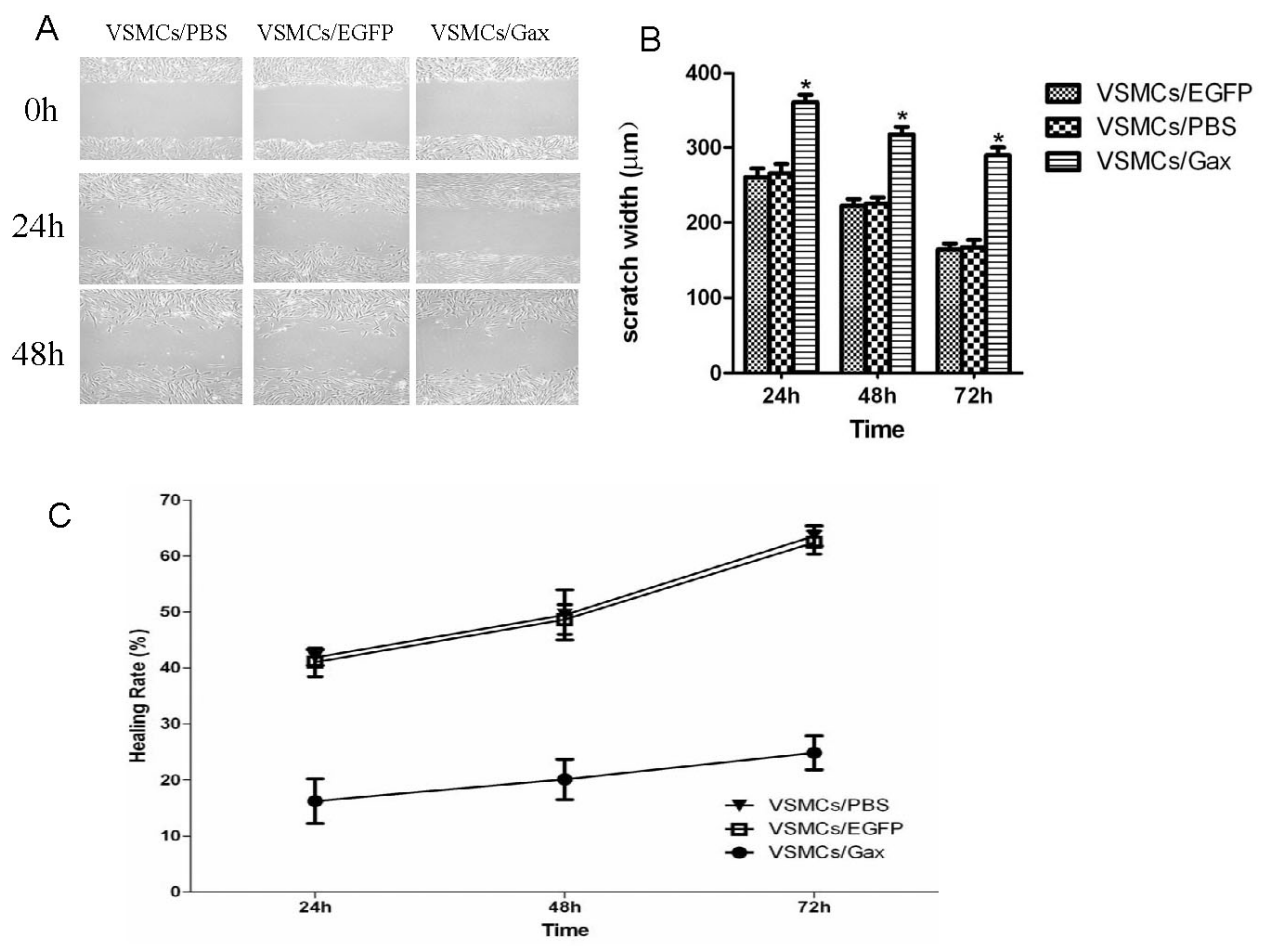

Figure 5. Results of migration assay in serum-induce VSMCs. VSMCs were seeded to $90 \%$ confluence on premarked 6-well plates. A central linear wound was scratched with a sterile pipet tip. Phase micrographs of scratch width were taken at $0,24,48$, and $72 \mathrm{~h}$. The photographs were analyzed by measuring the scratch width. Each experiment was tested in triplicate. A. VSMCs/Gax showed reduced migration ability compared with control groups. B. The scratch width was observed in VSMCs/Gax, VSMCs/EGFP and VSMCs/PBS. $* \mathrm{P}<0.05 v s$ VSMCs/ EGFP or VSMCs/PBS. C. Healing rate curves of serum-induced VSMCs was observed in different group.

\section{Effect of Gax overexpression on cell cycle control in serum-induced VSMCs}

Flow cytometry with propidium-iodide labeling was conducted to determine the effect of Gax overexpression on cell cycle distribution in serum-induced VSMCs. As shown in Table 1 and Figure 6, VSMC/Gax displayed a significant increase in the number of G0/G1-phase cell populations with $66.65 \% \pm 3.75 \%$ cells in G0/G1 phase. In contrast, VSMC/EGFP and VSMC/PBS had fewer 
cells in G0/G1-phase ( $42.57 \pm 2.45 \%$ and $44.17 \pm 2.05 \%$, respectively) and there was no significant difference between them. The data indicated that Gax overexpression suppresses cell cycle arrest at the G0/G1-phase in serum-induced VSMCs. These observations suggest that Gax overexpression inhibits serum-induced VSMCs proliferation by inducing a specific block in cell cycle progression.

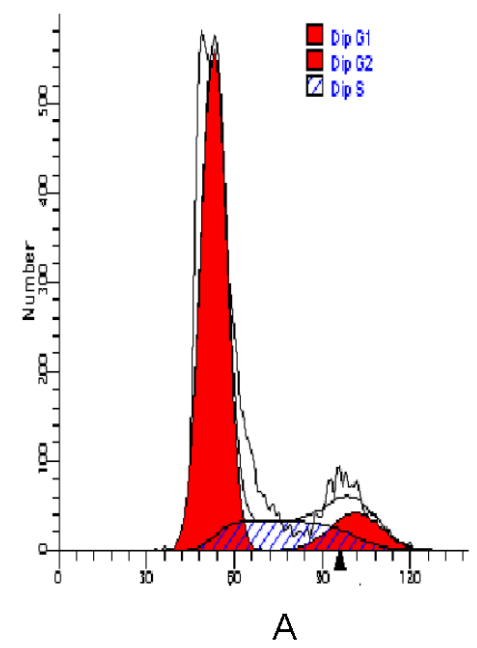

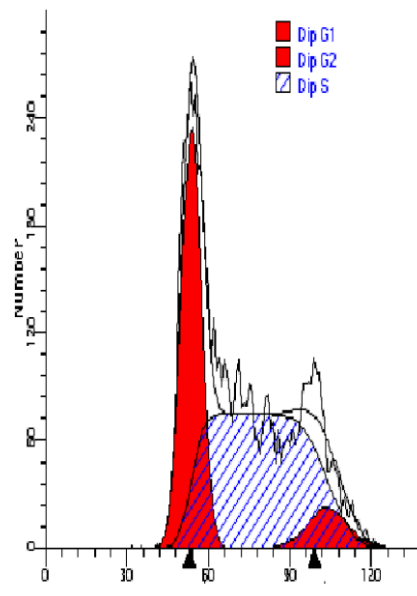

$\mathrm{B}$

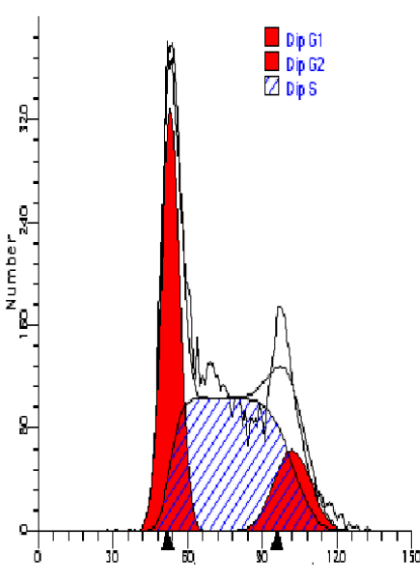

C

Figure 6. Cell cycle distribution analysis by flow cytometry in different groups. A. VSMCs/Gax; B. VSMCs/ EGFP; C. VSMCs/PBS.

Table 1. Cell cycle analysis by flow cytometry in serum-induced VSMCs from different groups.

\begin{tabular}{lcc}
\hline \multirow{2}{*}{ Group } & \multicolumn{2}{c}{ Cell cycle phase } \\
\cline { 2 - 3 } & G0/G1 (\%) & G2/M+S (\%) \\
\hline VSMCs/Gax & $66.65 \pm 3.75^{\mathrm{a}}$ & $33.35 \pm 3.75^{\mathrm{a}}$ \\
VSMCs/EGFP & $42.57 \pm 2.45$ & $57.43 \pm 2.45$ \\
VSMCs/PBS & $44.17 \pm 2.05$ & $55.83 \pm 2.05$ \\
\hline
\end{tabular}

${ }^{\mathrm{a}} \mathrm{P}<0.05, \mathrm{VSMCs} / \mathrm{Ad} 5-$ Gax group $v s$ VSMCs/EGFP or VSMCs/PBS.

\section{Effect of Gax overexpression on apoptosis in serum-induced VSMCs}

To determine whether Gax-overexpression induces apoptosis in serum-stimulated VSMCs, flow cytometry analyses and DAPI staining were performed. Cell apoptosis profiles were measured by flow cytometry and resulted in the following observations: The rate of apoptosis in VSMC/Gax was $13.12 \pm 0.97 \%$, which was significantly higher than those of VSMC/ EGFP $(7.86 \pm 1.26 \%)$ and VSMC/PBS $(5.94 \pm 0.80 \%)$ (Figure $7 ; \mathrm{P}<0.05)$, and there was no significant difference between VSMC/EGFP and VSMC/PBS. Similarly, DAPI staining showed that Ad5-Gax-transfected VSMCs had a higher apoptotic rate $(14.60 \pm 2.6 \%)$ than VSMCs transfected with Ad5-EGFP (7.40 $\pm 1.5 \%)$ or PBS (5.6 $\pm 1.0 \%)$. Furthermore, Gax overexpression-induced apoptosis in serum-induced VSMC/Gax cells resulted in apoptotic morphology features, such as cell shrinkage, chromatin compaction, and deep-staining nuclei. 
DAPI staining indicated marked morphological changes in nuclear chromatin in these cells. In the control groups, nuclear staining resulted in a less bright blue color that was more homogeneous. In contrast, condensed chromatin could be observed in VSMC/Gax cells (Figure 8). These data indicated that Gax overexpression induces apoptosis in serum-stimulated VSMCs.

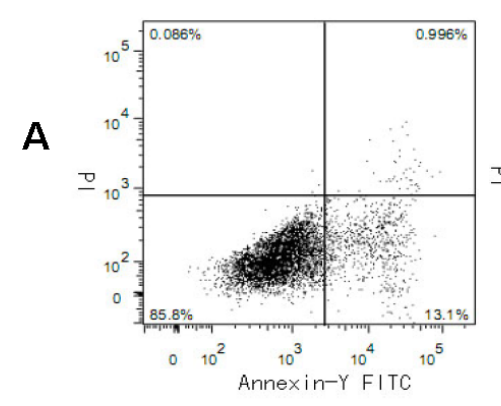

VSMCs/Ad5-Gax

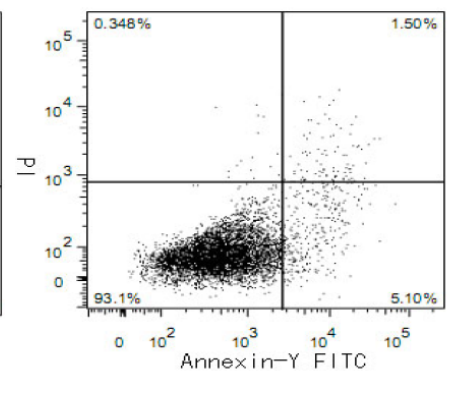

VSMCs/Ad5-EGFP

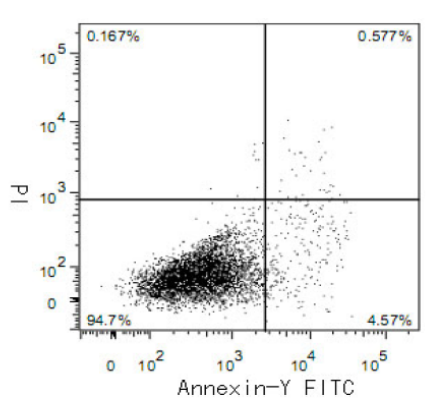

VSMCs/PBS

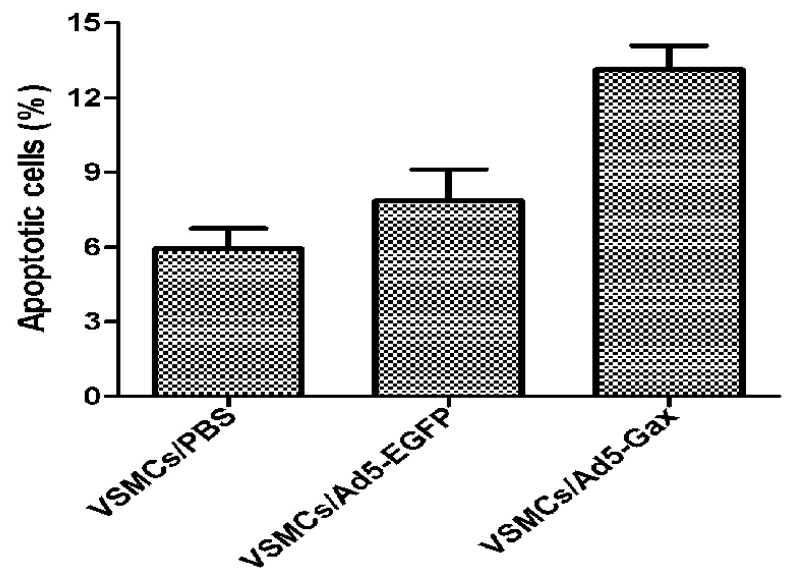

Figure 7. Results of serum-induced VSMCs apoptosis assay. Apoptosis assay in VSMCs/Ad5-Gax, VSMCs/Ad5EGFP, and VSMCs/PBS groups was examined using flow cytometry. (A) The typical flow cytometry results were exhibited for the three groups. Numbers in the superior and inferior quadrant on the right side represented the apoptotic percentage of cells for each. (B) The mean VSMCs apoptotic rates of three groups were shown. $\mathrm{P}<0.05$ for VSMCs/Ad5-Gax group vs VSMCs/Ad5-EGFP group or VSMCs/PBS group.
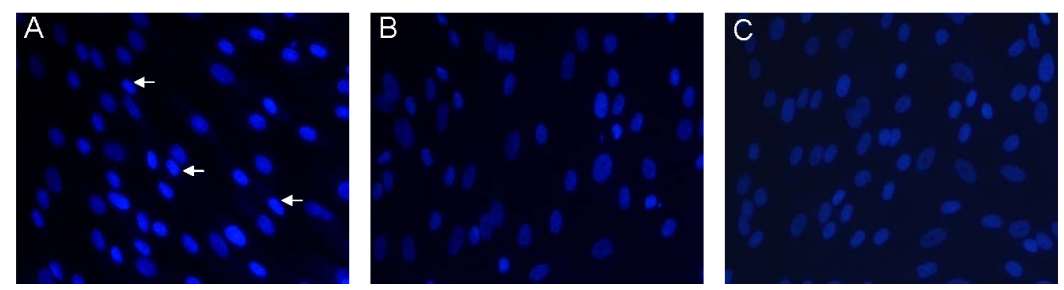

Figure 8. Serum-induced VSMCs apoptosis assay by DAPI staining. VSMCs were transfected with Ad5-Gax, Ad5EGFP or PBS. After that, the cells were stained with DAPI solution and reviewed under a fluorescent microscopy. Cells undergoing apoptosis are indicated by arrows. A. VSMCs/Gax; B. VSMCs/EGFP; C. VSMCs/PBS. 


\section{Detection of PCNA expression in serum-induced, Gax-overexpressing VSMCs}

To examine the effects of Gax overexpression on cell proliferation, we analyzed the cell proliferation-specific marker PCNA by using immunocytochemistry. As shown in Figure 9, the percentage of cells that were PCNA positive in VSMC/Gax was $23.12 \pm 6.97 \%$, significantly lower than that of VSMC/EGFP $(70.65 \pm 10.23 \%)$ and VSMC/PBS $(73.14 \pm 9.82 \%)(\mathrm{P}$ $<0.05)$, with no significant differences between the latter two treatments. It is suggested that Gax overexpression could reduce proliferation of serum-stimulated VSMCs.

\section{a}

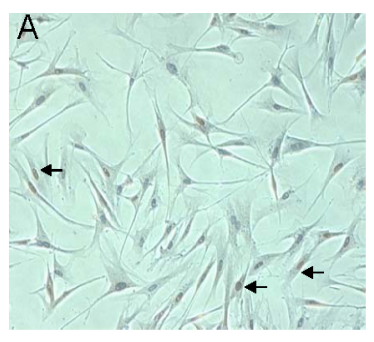

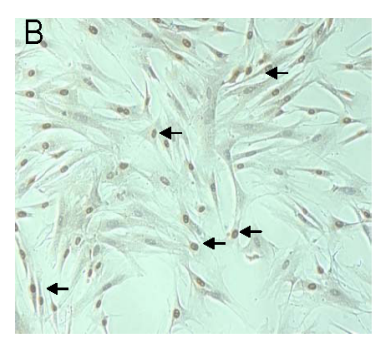

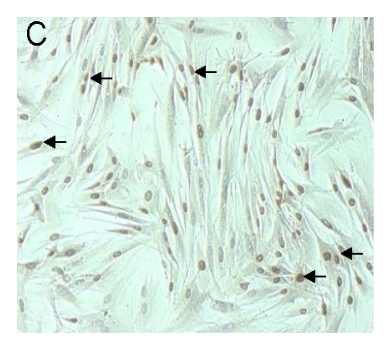

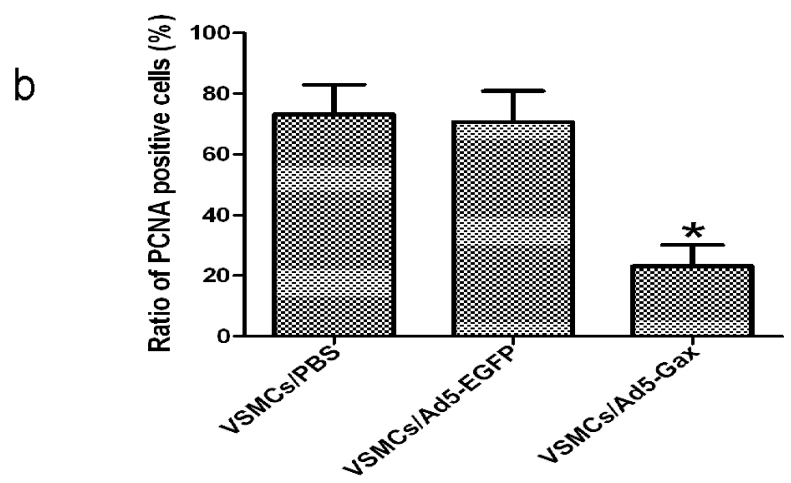

Figure 9. Results of PCNA immunohistochemistry staining. VSMCs were seeded in 6-wells plate and transfected with Ad5-Gax, Ad5-EGFP or PBS. After that, the cells were undergoing immunohistochemical staining of PCNA and reviewed under a microscopy. a. The typical immunohistochemistry graphs were exhibited for three groups. PCNA positive VSMCs were indicated by arrows. A. VSMCs/Gax; B. VSMCs/EGFP; C. VSMCs/PBS. b. The ratio of PCNA positive VSMCs in three groups were shown. $* \mathrm{P}<0.05$ vs VSMCs/EGFP or VSMCs/PBS.

\section{Effect of Gax overexpression on MMP-2 in serum-induced VSMCs}

MMPs play an important role in cells migration. Because MMP-2 is constitutively expressed in VSMCs, we examined the effect of Gax overexpression on the expression and activity of MMP-2 by RT-PCR and gel zymography, respectively. As shown in Figure 10, compared with VSMC/EGFP and VSMC/PBS cells, expression of MMP-2 in VSMC/Gax was significantly decreased $(\mathrm{P}<0.05)$. VSMC/EGFP and VSMCs/PBS showed no significant differences in MMP-2 mRNA expression levels. Moreover, VSMC/Gax decreased the activated 
MMP-2 band compared with VSMCs/EGFP and VSMCs/PBS. These results suggest that Gax overexpression could reduce $M M P-2$ expression and inhibit MMP-2 protein activity.

A

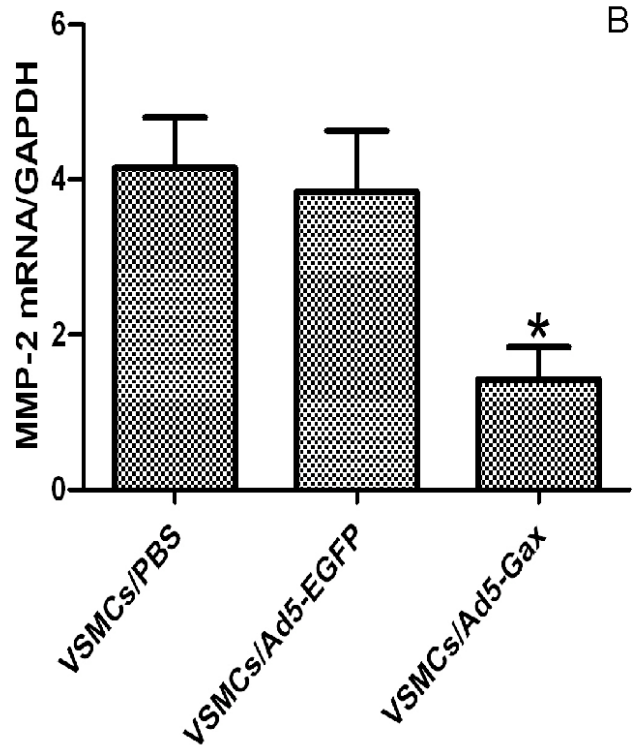

B

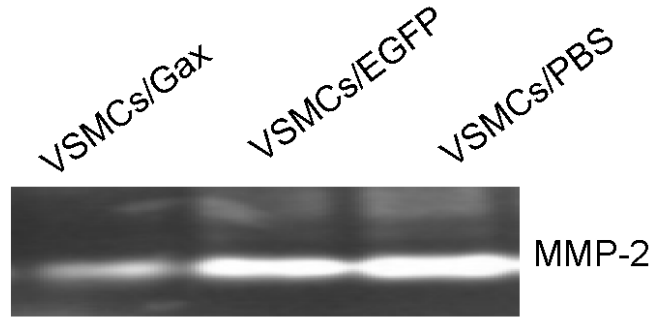

Figure 10. Effect of Gax overexpression on MMP-2 in serum-induced VSMCs. VSMCs were cultured in plates, followed by incubation with Ad5-Gax, Ad5-EGFP or PBS. Then the MMP-2 expression in each group was analyzed by RT-PCR and Gelatin zymography, respectively. A. Statistical analyses of the effect of Gax on release of MMP-2 in serum-induced VSMCs in terms of transcriptical level, GAPDH was a internal control. $* \mathrm{P}<0.05 v s$ VSMCs/ EGFP or VSMCs/PBS. B. Representative gelatin zymograms showing that Gax overexpression inhibted MMP-2 activity in VSMCs under serum stimulation.

\section{DISCUSSION}

Vein graft restenosis is the main factor limiting the success of CABG. The development and progression of vein graft failure involves multistage processes. Since the early 1990 s, gene therapy has become a powerful preclinical tool for studying interventions in cardiovascular diseases. Moreover, vein grafts are ideally suited for gene therapy because they can be genetically manipulated ex vivo during a brief clinical period (approx. 30-60 min) before grafting into the coronary vasculature. However, to successfully prevent vein graft failure, suitable gene-delivery vectors and efficient therapeutic transgenes are required. Many candidate gene-delivery systems for vein grafts are available, including viral and non-viral systems. The majority of studies of overexpressing candidate therapeutic genes in vein grafts have demonstrated that adenovirus vector is the most suitable system because of its simplicity and effectiveness (Baker et al., 2006; Bhardwaj et al., 2008).

Here, we have constructed a recombinant Gax-containing adenoviral vector, which was successfully transfected into VSMCs derived from rabbit thoracic aorta, resulting in stable expression of exogenous Gax. Furthermore, Gax overexpression inhibited the proliferation of serum-induced VSMCs by blocking the transition from G1- to S-phase and promoting cell apoptosis. It is also shown that Gax overexpression decreases cell migration by inhibiting the 
expression and activity of MMP-2. These results provide a new experimental avenue involving Gax overexpression to suppress neointimal hyperplasia in vein graft failure.

The Gax gene, first isolated from adult rat aorta cDNA (Gorski et al., 1994), is a well-characterized member of the homeobox gene family and transcription factor. Gax is expressed primarily in cardiovascular tissues (Gorski and Walsh, 2000). During responses to platelet-derived growth factor (PDGF) in vitro and carotid artery injury in vivo, Gax is rapidly downregulated in VSMCs, whereas Gax is upregulated under conditions that favor cell-cycle arrest and contractile phenotypes in VSMCs (Gorski et al., 1993; Gorski and Walsh, 1995; Weir et al., 1995; Yamashita et al., 1997). In addition, Ang-II inhibits VSMCs growth via redox-sensitive mitogen-activated protein kinase (MAPK) activation that downregulates Gax expression (Saito et al., 2005). Gax expression is decreased during conversion into synthetic VSMCs with abnormal proliferative and migratory activities (Markmann et al., 2003; Han et al., 2006). Recent studies have demonstrated that Gax overexpression decreases the migration of vascular endothelium cells (Patel et al., 2005). However, few studies have investigated the effects of Gax overexpression on the biological behaviors of serum-induced VSMCs (Maillard et al., 1997; Smith et al., 1997; Zeng et al., 2006).

The abnormal proliferation of VSMCs has an important role in neointimal hyperplasia of vein grafts restenosis. Abnormal tissue growth depends on the delicate balance between cell proliferation and apoptosis. It is reported that after carotid arteries injury, VSMCs proliferation in the intima was highest at the 7th day postinjury, whereas the rate of apoptosis in VSMCs was unchanged and lower than $1 \%$, suggesting an insufficient rate of cell apoptosis concomitant with intimal hyperplasia (Kamenz et al., 2000). It has been demonstrated that increasing VSMCs apoptosis could decrease neointimal hyperplasia in vivo (Lim et al., 2006). Gax overexpression inhibits the proliferation of hypoxia-induced pulmonary arterial smooth muscle cells (PASMCs) and promotes apoptosis in the PASMCs (Xia et al., 2011). A functional screen for regulators of CKDN2A in fibroblasts revealed that Gax could directly bind the INK4a promoter and induce INK4a-dependent cell apoptosis (Irelan et al., 2009). In addition, increased evidence indicates that inhibiting cell cycle process could reduce cell proliferation. For example, it has been shown that Gax inhibits cell-cycle progression in vascular endothelial cells by activating the cyclin kinase inhibitor $\mathrm{p} 21^{\mathrm{WAF} 1 / \mathrm{CIP} 1}$ through direct interactions with upstream AT-rich sequences (Chen et al., 2007). In addition, Gax binding to RING finger protein 10 (RNF10) enhances the activation of the p $21^{\mathrm{WAF} / \mathrm{CIP} 1}$ promoter (Liu et al., 2005). In this study, the expression levels of Gax mRNA and protein in the experimental and control groups were detected by RT-PCR and immunofluorescence. Our results showed that expression of Gax mRNA and protein in VSMC/Gax cells was significantly higher than in the control cells. The results of the MTT assay, PCNA immunocytochemistry, flow cytometry, and DAPI staining indicated that VSMC/Gax cells were blocked in the G0/G1 phase, showed increased apoptosis, and displayed markedly decreased proliferation $(\mathrm{P}<0.05)$. Our study also showed that Gax overexpression inhibits proliferation in serum-induced VSMCs by blocking progression of the cell cycle process and by inducing cell apoptosis.

VSMCs migration is also involved in the pathological process of vein grafts restenosis. It has been reported that Gax overexpression inhibits the migration of adventitial fibroblasts cells via interactions with multiple inflammatory cytokines (Liu et al., 2010). Several studies have shown that the expression level of VNR integrins, including $\alpha \mathrm{v} \beta 3$, is increased in migrating cells (Brooks et al., 1994; Clark et al., 1996) and that Gax specifically downregulates $\beta 3$ and $\beta 5$ expression in VSMCs in vivo and in vitro (Witzenbichler et al., 1999), suggesting that the cell migration is regu- 
lated by Gax via integrins. In addition, MMPs are extracellular matrix-degrading enzymes, which also participate in cell migration, and MMP-2 is secreted constitutively from VSMCs. It has been shown that MMP-2 mediates the migration of isolated VSMCs through a basement membrane barrier (Pauly et al., 1994). In the mouse carotid ligation model, a MMP-2 knockout decreased VSMCs migration and intima formation (Johnson and Galis, 2004). Furthermore, chemokine-induced chemotaxis of VSMCs also depended on MMP-2 activity (Haque et al., 2004). In this study, the effect of overexpression Gax on serum-induced VSMCs was examined in a wound-healing assay, which showed that Gax overexpression could significantly inhibit migration of serum-induced VSMCs. Moreover, RT-PCR detected expression of the $M M P-2$ gene, and the results of our zymography assay showed that Gax overexpression also decreased the expression and activity of MMP-2 protein. Accordingly, our data suggest that Gax overexpression inhibits migration of serum-induced VSMCs by decreasing MMP-2 expression and activity (Gorski et al., 1993).

In conclusion, Gax could be overexpressed in VSMCs by using the Ad5-Gax vector. Gax overexpression decreased cell proliferation and migration, promoted cell apoptosis, blocked cell cycle progression, and inhibited MMP-2 release and activity in serum-stimulated VSMCs. The results of this study could help improve our understanding of whether the Gax gene is an optimal therapeutic transgene for gene therapy of vein graft failure.

\section{ACKNOWLEDGMENTS}

Study supported by grants from the youth project of the Shanghai Natural Science Fund (\#12ZR1446300) and the Technological Fund Project of Shanghai Jiao Tong University, School of Medicine (\#12XJ10013).

\section{REFERENCES}

Baker AH, Yim AP and Wan S (2006). Opportunities for gene therapy in preventing vein graft failure after coronary artery bypass surgery. Diabetes Obes. Metab. 8: 119-124.

Bhardwaj S, Roy H and Yla-Herttuala S (2008). Gene therapy to prevent occlusion of venous bypass grafts. Expert. Rev. Cardiovasc. Ther. 6: 641-652.

Brooks PC, Clark RA and Cheresh DA (1994). Requirement of vascular integrin alpha v beta 3 for angiogenesis. Science 264: 569-571.

Candia AF and Wright CV (1996). Differential localization of Mox-1 and Mox-2 proteins indicates distinct roles during development. Int. J. Dev. Biol. 40: 1179-1184.

Chen Y, Leal AD, Patel S and Gorski DH (2007). The homeobox gene GAX activates p21WAF1/CIP1 expression in vascular endothelial cells through direct interaction with upstream AT-rich sequences. J. Biol. Chem. 282: 507-517.

Clark RA, Tonnesen MG, Gailit J and Cheresh DA (1996). Transient functional expression of alphaVbeta 3 on vascular cells during wound repair. Am. J. Pathol. 148: 1407-1421.

Csoka AB, English SB, Simkevich CP, Ginzinger DG, et al. (2004). Genome-scale expression profiling of HutchinsonGilford progeria syndrome reveals widespread transcriptional misregulation leading to mesodermal/mesenchymal defects and accelerated atherosclerosis. Aging Cell 3: 235-243.

Fitzgibbon GM, Kafka HP, Leach AJ, Keon WJ, et al. (1996). Coronary bypass graft fate and patient outcome: angiographic follow-up of 5,065 grafts related to survival and reoperation in 1,388 patients during 25 years. J. Am. Coll. Cardiol. 28: 616-626.

Gorski DH, LePage DF, Patel CV, Copeland NG, et al. (1993). Molecular cloning of a diverged homeobox gene that is rapidly down-regulated during the G0/G1 transition in vascular smooth muscle cells. Mol. Cell Biol. 13: 3722-3733.

Gorski DH, LePage DF and Walsh K (1994). Cloning and sequence analysis of homeobox transcription factor cDNAs with an inosine-containing probe. Biotechniques 16: 856-2, 865.

Gorski DH and Walsh K (1995). Mitogen-responsive nuclear factors that mediate growth control signals in vascular myocytes. Cardiovasc. Res. 30: 585-592. 
Gorski DH and Walsh K (2000). The role of homeobox genes in vascular remodeling and angiogenesis. Circ. Res. 87: 865-872. Gorski DH and Leal AJ (2003). Inhibition of endothelial cell activation by the homeobox gene Gax. J. Surg. Res. 111: 91-99.

Han M, Wen JK, Zheng B, Cheng Y, et al. (2006). Serum deprivation results in redifferentiation of human umbilical vascular smooth muscle cells. Am. J. Physiol. Cell Physiol. 291: C50-C58.

Haque NS, Fallon JT, Pan JJ, Taubman MB, et al. (2004). Chemokine receptor-8 (CCR8) mediates human vascular smooth muscle cell chemotaxis and metalloproteinase-2 secretion. Blood 103: 1296-1304.

Irelan JT, Gutierrez Del AA, Gutierrez A, Peters G, et al. (2009). A functional screen for regulators of CKDN2A reveals MEOX2 as a transcriptional activator of INK4a. PLoS One 4: e5067.

Johnson C and Galis ZS (2004). Matrix metalloproteinase-2 and -9 differentially regulate smooth muscle cell migration and cell-mediated collagen organization. Arterioscler. Thromb. Vasc. Biol. 24: 54-60.

Kamenz J, Seibold W, Wohlfrom M, Hanke S, et al. (2000). Incidence of intimal proliferation and apoptosis following balloon angioplasty in an atherosclerotic rabbit model. Cardiovasc. Res. 45: 766-776.

Li Q and Ding J (2007). Gene expression analysis reveals that formation of the mouse anterior secondary palate involves recruitment of cells from the posterior side. Int. J. Dev. Biol. 51: 167-172.

Lim S, Jin CJ, Kim M, Chung SS, et al. (2006). PPARgamma gene transfer sustains apoptosis, inhibits vascular smooth muscle cell proliferation, and reduces neointima formation after balloon injury in rats. Arterioscler. Thromb. Vasc. Biol. 26: 808-813.

Lin J, Friesen MT, Bocangel P, Cheung D, et al. (2005). Characterization of Mesenchyme Homeobox 2 (MEOX2) transcription factor binding to RING finger protein 10. Mol. Cell. Biochem. 275: 75-84.

Liu P, Zhang C, Feng JB, Zhao YX, et al. (2008). Cross talk among Smad, MAPK, and integrin signaling pathways enhances adventitial fibroblast functions activated by transforming growth factor-betal and inhibited by Gax. Arterioscler. Thromb. Vasc. Biol. 28: 725-731.

Liu P, Zhang C, Zhao YX, Feng JB, et al. (2010). Gax gene transfer inhibits vascular remodeling induced by adventitial inflammation in rabbits. Atherosclerosis 212: 398-405.

Maillard L, Van BE, Smith RC, Le RA, et al. (1997). Percutaneous delivery of the gax gene inhibits vessel stenosis in a rabbit model of balloon angioplasty. Cardiovasc. Res. 35: 536-546.

Markmann A, Rauterberg J, Vischer P, Robenek H, et al. (2003). Expression of transcription factors and matrix genes in response to serum stimulus in vascular smooth muscle cells. Eur. J. Cell Biol. 82: 119-129.

Mitra AK, Gangahar DM and Agrawal DK (2006). Cellular, molecular and immunological mechanisms in the pathophysiology of vein graft intimal hyperplasia. Immunol. Cell Biol. 84: 115-124.

Patel S, Leal AD and Gorski DH (2005). The homeobox gene Gax inhibits angiogenesis through inhibition of nuclear factor-kappaB-dependent endothelial cell gene expression. Cancer Res. 65: 1414-1424.

Pauly RR, Passaniti A, Bilato C, Monticone R, et al. (1994). Migration of cultured vascular smooth muscle cells through a basement membrane barrier requires type IV collagenase activity and is inhibited by cellular differentiation. Circ. Res. 75: 41-54.

Saito T, Itoh H, Yamashita J, Doi K, et al. (2005). Angiotensin II suppresses growth arrest specific homeobox (Gax) expression via redox-sensitive mitogen-activated protein kinase (MAPK). Regul. Pept. 127: 159-167.

Skopicki HA, Lyons GE, Schatteman G, Smith RC, et al. (1997). Embryonic expression of the Gax homeodomain protein in cardiac, smooth, and skeletal muscle. Circ. Res. 80: 452-462.

Smith RC, Branellec D, Gorski DH, Guo K, et al. (1997). p21CIP1-mediated inhibition of cell proliferation by overexpression of the gax homeodomain gene. Genes Dev. 11: 1674-1689.

Veith FJ, Gupta SK, Ascer E, White-Flores S, et al. (1986). Six-year prospective multicenter randomized comparison of autologous saphenous vein and expanded polytetrafuoroethylene grafts in infrainguinal arterial reconstructions. $J$. Vasc. Surg. 3: 104-14.

Weir L, Chen D, Pastore C, Isner JM, et al. (1995). Expression of gax, a growth arrest homeobox gene, is rapidly downregulated in the rat carotid artery during the proliferative response to balloon injury. J. Biol. Chem. 270: 5457-5461.

Witzenbichler B, Kureishi Y, Luo Z, Le RA, et al. (1999). Regulation of smooth muscle cell migration and integrin expression by the Gax transcription factor. J. Clin. Invest. 104: 1469-1480.

Xia S, Tai X, Dong J, Qian G, et al. (2007). [Effect of Ad-Gax transfection on apoptosis of human lung adenocarcinoma A549 cells and its mechanism]. Zhongguo Fei. Ai. Za Zhi. 10: 269-274.

Xia S, Tai X, Wang Y, An X, et al. (2011). Involvement of Gax gene in hypoxia-induced pulmonary hypertension, proliferation, and apoptosis of arterial smooth muscle cells. Am. J. Respir. Cell Mol. Biol. 44: 66-73.

Yamashita J, Itoh H, Ogawa Y, Tamura N, et al. (1997). Opposite regulation of Gax homeobox expression by angiotensin II and C-type natriuretic peptide. Hypertension 29: 381-387.

Zeng JH, Yang Z, Xu J, Qiu ML, et al. (2006). Down-regulation of the gax gene in smooth muscle cells of the splenic vein of portal hypertension patients. Hepatobiliary. Pancreat. Dis. Int. 5: 242-245. 